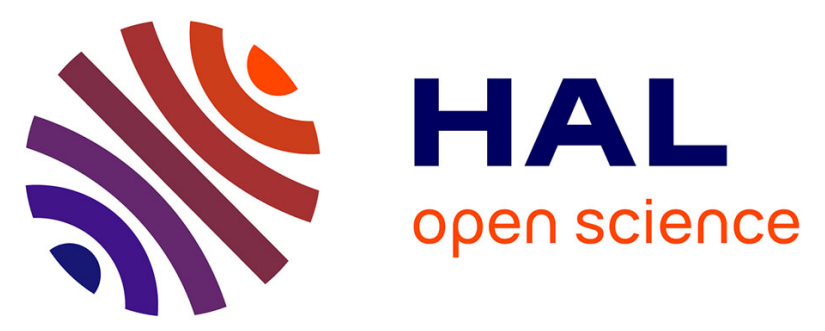

\title{
A nonlinear macroscopic multi-phasic model for describing interactions between solid, fluid and ionic species in biological tissue materials
}

Long-Yuan Li, Peter Pinsky

\section{- To cite this version:}

Long-Yuan Li, Peter Pinsky. A nonlinear macroscopic multi-phasic model for describing interactions between solid, fluid and ionic species in biological tissue materials. Philosophical Magazine, 2010, 91 (2), pp.311. 10.1080/14786435.2010.519353 . hal-00631266

\author{
HAL Id: hal-00631266 \\ https://hal.science/hal-00631266
}

Submitted on 12 Oct 2011

HAL is a multi-disciplinary open access archive for the deposit and dissemination of scientific research documents, whether they are published or not. The documents may come from teaching and research institutions in France or abroad, or from public or private research centers.
L'archive ouverte pluridisciplinaire HAL, est destinée au dépôt et à la diffusion de documents scientifiques de niveau recherche, publiés ou non, émanant des établissements d'enseignement et de recherche français ou étrangers, des laboratoires publics ou privés. 


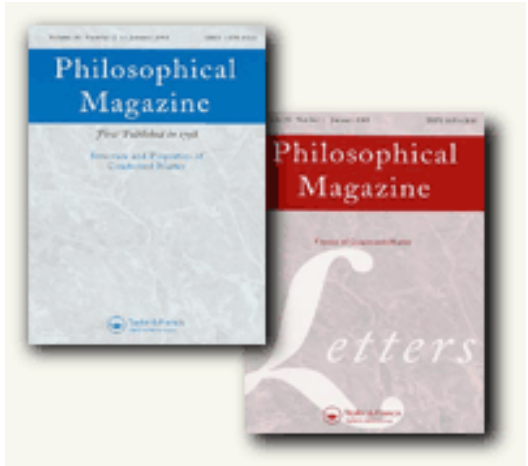

\section{A nonlinear macroscopic multi-phasic model for describing interactions between solid, fluid and ionic species in biological tissue materials}

\begin{tabular}{|r|l|}
\hline Journal: & Philosophical Magazine \& Philosophical Magazine Letters \\
\hline Manuscript ID: & TPHM-10-Apr-0149.R1 \\
\hline Journal Selection: & Philosophical Magazine \\
\hline Date Submitted by the \\
Author: & 02-Jul-2010 \\
\hline Complete List of Authors: & $\begin{array}{l}\text { Li, Long-yuan; University of Birmingham, Civil Engineering } \\
\text { Pinsky, Peter; Stanford University, Mechanical Engineering }\end{array}$ \\
\hline Keywords: & $\begin{array}{l}\text { anisotropic elasticity, biological materials, computer modelling, } \\
\text { diffusion, elasticity, ionic transport, porous media }\end{array}$ \\
\hline Keywords (user supplied): & \\
\hline
\end{tabular}

\section{s) ScholarONE" \\ Manuscript Central}




\begin{abstract}
This paper presents a nonlinear, macroscopic multi-phasic model for describing the interactions between solid, fluid, and ionic species taking place in porous materials. Governing equations are derived based on the nonlinear theories of solid mechanics, linear flow theory of Newtonian fluids, and theory of irreversible thermodynamics for the transport of ions and ionic solutions. The model shows that the transport coupling between ions and ionic solution exists only when the porous material has a membrane-like feature which could be inside the material or on the material boundaries. Otherwise, the coupling occurs only between the solid and fluid phases and the transport of ionic species will have no effect on the macroscopic stresses, strains and displacements of the porous material. As an application of the present multi-phasic model, a numerical example of the human cornea under the shock of $\mathrm{NaCl}$ hypertonic solution applied to its endothelial surface is presented. This is a typical example of how ionic transport induces swelling in biological tissues. The results obtained from the present multi-phasic model demonstrate that the mechanical properties of the tissue have important influence on the swelling of the cornea. Without taking into account this influence, the predicted swelling may be exaggerated.
\end{abstract}

Keywords - porous media, nonlinear, multi-phasic, ionic transport, corneal swelling

\title{
1. Introduction
}

Multiphysics problems involving fluid-solid, chemical-fluid-solid, and electrochemical-fluid-solid interactions often arise in porous materials. A porous material is a solid (often called the matrix or skeleton) permeated by an interconnected network of pores (voids) filled with a fluid which may be a pure solvent or an ionic solution. Usually both solid matrix and the pore network (also known as the pore space) are assumed to be continuous, so as to form two interpenetrating continua such as in a sponge. Many natural substances such as rocks, soils, biological tissues, and manmade materials such as foams and ceramics can be considered to be porous materials. Porous materials whose solid matrix is elastic with a viscous fluid occupying the pores are referred to as poroelastic materials. A poroelastic material is characterized by its porosity and permeability, as well as the properties of its constituents (solid matrix and fluid).

Poroelasticity theory describes the interaction between the flow and deformation in porous materials. The concept of a porous material originally emerged in soil mechanics, and in particular in the works of Terzaghi (1923), who introduced the concept of effective stress to describe the equilibrium problem of consolidation in soil mechanics. However a more general concept of a poroelastic material, independent of its nature or application, is attributed to Biot $(1941,1957,1973)$. The Biot theory of 
poroelasticity provides a complete and general description of the mechanical behaviour of poroelastic materials, which has been widely used in civil, mining and petroleum engineering, acoustic wave propagation in saturated media and recently in biology and biomedical engineering.

Biot's equations for the linear theory of poroelasticity are derived from equations of linear elasticity for the solid matrix together with Darcy's law for the flow of fluid through the porous matrix. Since the early 1980s, interest has intensified concerning the role of poroelasticity on the mechanical behaviour of biological tissues. Several models have been proposed to investigate the biomechanics of soft tissues based on Biot's poroelastic theory. They include, among others, successful applications in the study of articular cartilage (Mow et al., 1980, 1984), cardiac muscle (Yang and Taber, 1991), arterial walls (Simon et al., 1993, 1998), skin and bones (Mak et al., 1994, 2001). All these models have been based on the concept of tissues as structurally biphasic, i.e. composed of a solid matrix saturated by an interstitial fluid. By incorporating material nonlinearity of the solid matrix into the biphasic framework, the model has been able to describe the nonlinear response of porous materials (Holmes, 1986; Cohen et al., 1998; Soulhat et al., 1999; Soltz and Ateshian, 2000).

For most biological tissues, the fluid phase contains ionic species and the solid phase may also carry fixed electric charges. In some cases the spatial concentration gradient of ionic species in the fluid phase can generate significant osmotic pressure, which in magnitude may be comparable to the fluid pressure. In these cases the transport of ionic species in the fluid phase also needs to be considered in the model as the osmotic pressure can influence the fluid flow and thus the deformation of the porous material. Lai et al (1991) proposed a triphasic model of articular cartilage as an extension of the biphasic model, where negatively charged proteoglycans are modelled to be fixed to the solid matrix, and monovalent ions in the interstitial fluid are modelled as additional fluid phases. This model was later extended to incorporate multiple polyvalent ions by $\mathrm{Gu}$ et al. (1997,1999). Similar modification of the biphasic model to include thermo-chemo-electro-mechanical interactions in saturated charged porous solids was made by Huyghe and Janssen (1999), van Meerveld et al. (2003) and Zhang and Szeri (2007). Finite element analyses based on triphasic models were undertaken by Ferguson et al. (2004) and Snijders et al. (2005) for the prediction of biomechanical behaviour of intervertebral disc tissues and other swelling porous media.

In this paper, a nonlinear, macroscopic multi-phasic model is proposed for describing the interactions between solid, fluid, and ionic species taking place in porous materials. In contrast to existing multi-phasic models, the present model not only considers the geometrical and material nonlinearities of the porous material but also uses a more general flow theory describing the transport of ionic solution and ionic species in the medium with or without membranes. The model can be regarded as a direct extension of Biot's poroelasticity from the linear interaction between solid and fluid phases to the nonlinear interactions between solid, fluid and ionic species. The model can be used to predict the interaction between mechanical and physiochemical properties in biological tissues.

2. Governing equations in the biphasic model 
Consider a porous material that is made of a microscopically inhomogeneous solid matrix filled with a homogeneous fluid undergoing large displacements, which deforms from an initial undeformed configuration into the current deformed configuration (see Figure 1). Let $\Omega$ be a representative elementary volume of the porous material. The representative elementary volume is defined as being fixed to the continuous solid frame; hence the solid mass is conserved within the representative elementary volume when it deforms from $\Omega_{0}$ in the undeformed configuration to $\Omega$ in a deformed configuration. The fluid part of the representative elementary volume occupies the pore space enclosed within the solid frame, and its mass is not necessarily conserved.

Let $\Omega_{\mathrm{s}}$ and $\Omega_{\mathrm{f}}$ be the volumes occupied by the solid and fluid phases in $\Omega$, such that $\Omega=\Omega_{s} \cup \Omega_{f}$, and $\Omega_{\text {so }}$ and $\Omega_{\mathrm{fo}}$ be the volumes occupied by the solid and fluid phases in $\Omega_{o}$, such that $\Omega_{o}=\Omega_{s o} \cup \Omega_{f o}$. The external surfaces $\Gamma$ of the representative elementary volume $\Omega$ are the sum of the solid portion $\Gamma_{s}$ and the fluid portion $\Gamma_{f}$, such that $\Gamma=\Gamma_{s} \cup \Gamma_{f}$. The porosity $\phi$ of the representative elementary volume thus is defined as (Lopatnikov and Cheng, 2002)

$$
\phi=\frac{\Omega_{f}}{\Omega}=\frac{\Gamma_{f}}{\Gamma}
$$

Let $\mathbf{X}$ denote the position vector of a material point of the solid matrix in the porous material in the undeformed configuration and $\mathbf{x}=\varphi(\mathbf{X})$ represents the position vector of the material point $\mathbf{X}$ in the deformed configuration. The function $\varphi(\mathbf{X})$ is called the deformation mapping from the initial undeformed configuration to the current deformed configuration. By introducing the deformation gradient tensor $\mathbf{F}$, where

$$
\mathbf{F}=\frac{\partial \varphi}{\partial \mathbf{X}}
$$

the right and left Cauchy-Green tensors, $\mathbf{C}$ and $\mathbf{B}$, can be expressed in terms of the deformation gradient tensor as follows

$$
\mathbf{C}=\mathbf{F}^{T} \mathbf{F}, \mathbf{B}=\mathbf{F F}^{T}
$$

The displacement vector $\mathbf{u}$ of the material point $\mathbf{X}$ is given by the difference between its current position and its initial position, so

$$
\mathbf{u}(\mathbf{X})=\varphi(\mathbf{X})-\mathbf{X}=\mathbf{x}-\mathbf{X}
$$

The Green strain tensor $\mathbf{E}$ can be expressed in terms of the deformation gradient tensor or the displacement gradients as follows

$$
\mathbf{E}=\frac{1}{2}\left(\mathbf{F}^{T} \mathbf{F}-\mathbf{I}\right)=\frac{1}{2}(\mathbf{C}-\mathbf{I})=\frac{1}{2}\left[\left(\nabla_{o} \mathbf{u}\right)^{T}+\left(\nabla_{o} \mathbf{u}\right)+\left(\nabla_{o} \mathbf{u}\right)\left(\nabla_{o} \mathbf{u}\right)^{T}\right]
$$


where the operator $\nabla_{o}$ is the gradient with respect to the material coordinates and $\mathbf{I}$ is the second order unit tensor. It is clear from equations (2)-(5) that if the displacement vector at a material point is known then the deformation gradient tensor, the CauchyGreen tensors and the Green strain tensor at that material point can be determined.

The equilibrium of the porous material is expressed in terms of the total Cauchy stress tensor $\sigma$ by,

$$
\nabla \cdot \boldsymbol{\sigma}+\mathbf{b}=0 \quad \text { in } \Omega
$$

where $\mathbf{b}$ is the body force field per unit volume. Based on the assumption of Biot's poroelasticity, the constitutive equation for a fluid-filled porous material can be expressed as follows (Biot, 1957; Detournay and Cheng, 1993)

$$
\mathbf{S}-\mathbf{S}_{o}=\frac{\partial W}{\partial \mathbf{E}}-\left(\mathbf{F}^{-1} J \mathbf{F}^{-T}\right) \alpha p
$$

where $\mathbf{S}$ is the second Piola-Kirchhoff stress tensor, $\mathbf{S}_{\mathrm{o}}$ is the initial second PiolaKirchhoff stress tensor, $W$ is the strain energy density function of the porous material, $J=\operatorname{det}(\mathbf{F})$ is the Jacobian of the deformation gradient tensor $\mathbf{F}, \alpha$ is a material constant, and $p$ is the fluid pressure. Equation (7) is a generalization of the constitutive equation for linear and isotropic porous materials (Biot, 1957; Detournay and Cheng, 1993) suitable for nonlinear and anisotropic porous materials. The first and second terms in the right-hand-side of equation (7) represent the stress contributions from the solid matrix and fluid pressure, respectively. The factor in parenthesis is the pull-back of the fluid pressure through the motion to the reference configuration. For anisotropic porous materials, examples of the strain energy density function $\mathrm{W}$ can be found in Gasser et al. (2006) and Pinsky et al. (2005). The relationship between the total Cauchy stress tensor in equation (6) and the second Piola-Kirchhoff stress tensor in equation (7) is given by

$$
\boldsymbol{\sigma}=J^{-1} \mathbf{F S F}^{T}
$$

Equations (2)-(8) are similar to those describing the nonlinear elastic deformation of hyperelastic materials under externally applied loads. The only difference here is the constitutive equation (7) involves the fluid pressure. It is for this reason that additional equations are required in order to determine the stresses, strains and displacements. It should be emphasized that the displacements, strains and stresses used here are based on a macroscopic description and referenced to the porous material. Thus the deformations or strains used in the present formulation may also include the contribution from the change of porosity of the porous material, which is not properly addressed in Biot's theory.

Fluid pressure in a porous material depends not only on the microstructure of the porous material but also on the flow of the fluid through the connective pores. Fluid flow in porous materials has been studied for more than a century. The interaction between solid and liquid phases in porous materials was first quantified by Darcy in 1856. In a study of water percolating through sand, Darcy discovered that the flow rate was proportional to the pressure gradient. This empirical relationship was 
theoretically derived later by other investigators on the basis of mechanical analysis of fluid flow in porous materials (Bear, 1988; de Boer, 2000), which demonstrated that Darcy's law is valid for most Newtonian liquids at low flow velocity. Fluids in many biological materials and in most rock and soil materials belong to this category and therefore Darcy's law can be applied in these fields.

Let $\mathbf{q}$ be the specific discharge vector, which describes the motion of the fluid relative to the solid in a porous material, which is formally defined as the rate of fluid volume crossing unit area of the porous material, and $\zeta$ be the change of fluid content of the porous material with respect to the initial state, which is defined as the variation of fluid volume per unit volume of porous material due to permeable fluid mass transport. A positive $\zeta$ corresponds to a "gain" of fluid by the porous solid. The relationship between $\mathbf{q}$ and $\zeta$ can be obtained by considering the volumetric conservation of the fluid in the representative elementary volume as follows (Biot, 1957; Detournay and Cheng, 1993)

$$
\frac{\partial \zeta}{\partial t}=-\nabla_{o} \cdot \mathbf{q}
$$

where $t$ is time. The flow of fluid within the porous material can be described by Darcy's law. By ignoring the fluid density variation effect, Darcy's law can be simply expressed as follows

$$
\mathbf{q}=-\mathbf{K} \nabla p
$$

where $\mathbf{K}$ is the permeability tensor which may reduce to diagonal form if the porous material is orthotropic or isotropic. Substituting equation (10) into (9) yields,

$$
\frac{\partial \zeta}{\partial t}=\nabla_{o} \cdot(\mathbf{K} \nabla p)
$$

In addition to equation (11), an equation of state that links the fluid pressure to the volume deformations of the fluid phase and the porous material is needed in order that both the variation of fluid content and fluid pressure can be determined. The equation of state for a general porous material can be expressed as follows (Detournay and Cheng, 1993),

$$
p=M[\zeta-\alpha(J-1)]
$$

where $M$ and $\alpha$ are the material constants. For linear problems of small deformation $J$ $=1+\operatorname{Tr}(\mathrm{E})$ where $\operatorname{Tr}(\mathrm{E})$ is the volume dilatation of the porous material. Equations (11) and (12) provide two additional equations which together with equations (5)-(7) can be used to determine the stresses, strains, displacements, variation of fluid content and fluid pressure in the porous material.

For most biological materials, both the solid and fluid phases of the porous material can be regarded as incompressible. In this case the change of volume of the porous material is caused only by the "gain" or "loss" of the fluid, that is, $J-1=\zeta$. In this 
case the values of the material constants $M$ and $\alpha$ can be taken as $M \rightarrow \infty$ and $\alpha=1$ (Detournay and Cheng, 1993). Eliminating $\zeta$ from equations (12), equation (11) yields

$$
\frac{\partial J}{\partial t}=\nabla_{o} \cdot(\mathbf{K} \nabla p)
$$

Equations (5), (6), (7) and (13) are the governing equations for determining stresses, strains, displacements and fluid pressure in fluid-filled porous materials whose solid and fluid phases are incompressible. The porous material can be nonlinear and anisotropic elastic material. The boundary conditions for stresses and displacements are the same as those normally used in solid mechanics. The boundary conditions for the fluid pressure require that the pressure by prescribed if the boundary surface is permeable, otherwise a zero normal derivative of fluid pressure can be assumed. The governing equations involve the equilibrium, geometrical and constitutive equations of solid materials and the moment balance equation (Darcy's law) of fluids, and so the model is usually referred to as the biphasic model. The linear version of the present biphasic model has been proposed by Naili et al. (1998) and Swartz et al. (1999). However, the present model is more general as not only is it nonlinear but also anisotropic.

\section{Governing equations in the triphasic model}

For most biological materials, the fluid is an ionic solution and thus the transport in the porous material involves not only the flow of ionic solution but also the diffusion, convection and migration of ionic species. Dependent on the properties of the porous material, the permeability of a porous material may be different for the solvent and solutes. If so, osmotic pressure may be generated when the ionic species have concentration gradients. This osmotic pressure can affect the flow of solution and thus the overall deformation of the porous material.

The governing equations describing the transport of ionic solution and ionic species in porous materials can be derived in terms of the mass conservation of individual components in the representative elementary volume as follows

$$
\begin{aligned}
& \frac{\partial\left(\rho_{f} \Omega_{f} / \Omega_{o}\right)}{\partial t}=-\nabla_{o} \cdot\left(\rho_{f} \mathbf{q}\right) \\
& \frac{\partial\left(C_{k} \Omega_{f} / \Omega_{o}\right)}{\partial t}=-\nabla_{o} \cdot \mathbf{N}_{k}
\end{aligned}
$$

where $\rho_{f}$ is the density of the ionic solution, $C_{k}$ is the concentration of species $k$, and $\mathbf{N}_{\mathrm{k}}$ is the molar flux of species $k$ flowing in unit time through unit area of porous material. For a dilute solution $\rho_{f}$ can be taken as the density of the solvent and thus is a constant. Note that,

$$
\frac{\Omega_{f}}{\Omega_{o}}=\frac{\Omega_{f}-\Omega_{f o}}{\Omega_{o}}+\frac{\Omega_{f o}}{\Omega_{o}}=\zeta+\phi_{o}
$$


where $\phi_{o}=\Omega_{f o} / \Omega_{o}$ is the initial porosity of the porous material. It is clear from the relationship between $\zeta$ and $\Omega_{f}$ given by equation (16) that equation (14) is equivalent to equation (9) when $\rho_{f}$ is constant. However, for the porous material of incompressible solid and fluid phases, $\zeta$ can be replaced with $J-1$. Also, for a macroscopic model it is more convenient to use $\zeta$ than $\Omega_{f}$. Note that $\Omega_{s} \equiv \Omega_{s o}$ for a porous material with an incompressible solid phase. In this case the change of porosity can be calculated directly from the variation of fluid content $\zeta$ as follows

$$
\zeta=\frac{\Omega_{f}-\Omega_{f o}}{\Omega_{o}}=\phi \frac{\Omega}{\Omega_{o}}-\phi_{o}=\frac{\phi-\phi_{o}}{1-\phi}
$$

Note that equation (17) is slightly different from that proposed by $\mathrm{Gu}$ et al. (1993, 1999). In Gu et al. (1993, 1999), the volume dilatation is taken as the solid phase volume dilatation, whereas in the present formulation, it is taken as the porous matrix volume dilatation. It is believed that the present volume dilatation definition is more appropriate because the strains are calculated from the displacements which are the macroscopic average displacements of the porous material rather than the displacements of the solid phase of the porous material in the representative elementary volume.

The fluxes of the solution and solutes which appear in equations (14) and (15) can be expressed in two different ways. One is to use the common approach in which the flux of the solution is defined by Darcy's law, that is, equation (10), and that of the solutes is defined by the Nernst-Planck equation (Newman, 1991), that is,

$$
\mathbf{N}_{k}=-\mathbf{D}_{k} \nabla C_{k}-\left(\frac{z_{k} F}{R T}\right) C_{k} \mathbf{D}_{k} \nabla \Phi+C_{k} \mathbf{q}
$$

where $\mathbf{D}_{k}$ and $z_{k}$ are the diffusion tensor and charge number of species $k$, respectively, $F$ is the Faraday constant, $R$ is the universal gas constant, $T$ is the absolute temperature, and $\Phi$ is the electrostatic potential. For an isotropic porous material $\mathbf{D}_{k}$ reduces to diagonal form. The fluxes defined in equations (10) and (18) do not include those generated by the osmotic pressure and thus they can be applied to porous materials that do not have membrane-like feature, which has no osmotic pressure. The other is based on the theory of irreversible thermodynamics (Kedem and Katchalsky, 1958; Li, 2004) in which the fluxes of the solution and solutes across a permeable membrane are expressed as follows

$$
\begin{aligned}
& \mathbf{q}=-L_{p}\left[\Delta p-\sum_{k=1}^{M} \sigma_{k} R T\left(\Delta C_{k}+\frac{z_{k} F}{R T} \bar{C}_{k} \Delta \Phi\right)\right] \\
& \mathbf{N}_{k}=\left(1-\sigma_{k}\right) \bar{C}_{k} \mathbf{q}-\omega_{k} R T\left(\Delta C_{k}+\frac{z_{k} F}{R T} \bar{C}_{k} \Delta \Phi\right)
\end{aligned}
$$

where $L_{p}$ is the hydraulic conductivity coefficient, $\sigma_{k}$ and $\omega_{k}$ are the reflection coefficient and permeability coefficient of species $k$, respectively, $\Delta p, \Delta C_{k}$, and $\Delta \Phi$ are the difference of pressures, concentrations and electrostatic potentials in the 
solutions on each side of the membrane wall, $\bar{C}_{k}$ is the average concentration of species $k$ (see Figure 2), and $M$ is the total number of species involved in the solution. For a semipermeable membrane of thickness $h_{m}$, the gradient of a variable across the membrane can be approximated as $\nabla()=.\Delta(.) / h_{m}$. Thus equations (19) and (20) can be re-written as

$$
\begin{aligned}
& \mathbf{q}=-\overline{\mathbf{L}}_{p}\left[\nabla p-\sum_{k=1}^{M} \sigma_{k} R T\left(\nabla C_{k}+\frac{z_{k} F}{R T} C_{k} \nabla \Phi\right)\right] \\
& \mathbf{N}_{k}=\left(1-\sigma_{k}\right) C_{k} \mathbf{q}-\overline{\boldsymbol{\omega}}_{k} R T\left(\nabla C_{k}+\frac{z_{k} F}{R T} C_{k} \nabla \Phi\right)
\end{aligned}
$$

where $\overline{\mathbf{L}}_{p}=L_{p} h_{m} \mathbf{n}$ is the equivalent hydraulic conductivity coefficient matrix, $\overline{\boldsymbol{\omega}}_{k}=\omega_{k} h_{m} \mathbf{n}$ is the equivalent permeability coefficient matrix of species $k$, and $\mathbf{n}$ is the normal of the membrane. Equations (21) and (22) can be compared with Darcy's law (equation (10)) and the Nernst-Planck equation (equation (18)), where $\overline{\mathbf{L}}_{p}$ is equivalent to $\mathbf{K}$, and $\overline{\boldsymbol{\omega}}_{k} R T$ is equivalent to $\mathbf{D}_{k}$. The only difference between the two approaches is that in the Nernst-Planck case $\sigma_{k}=0$, while in the current approach it is not necessarily zero. Thus, the former can be regarded as a special case of the latter approach. For a porous material which has equal permeability to the solvent and solutes (i.e., there is no membrane-like feature) one can simply take $\sigma_{k}=0$. Equations (21) and (22) imply that a porous material with membrane-like feature can be regarded as an assembly of a large number of membranes.

Equations (21) and (22) show that the fluxes of solution and solutes are coupled. The first two terms in the right-hand-side of equation (21) represent the fluxes resulting from the gradients of fluid pressure and osmotic pressure, respectively. It is seen from equation (22) that the convective velocities of solutes are not necessarily equal to the solution velocity. This is because the permeability of the solvent and solutes in the porous material may be different. The solutes are normally more hindered by the pore surfaces and thus $0 \leq \sigma_{k} \leq 1$.

Note that for charged ions the molar fluxes of ionic species should satisfy the conservation equation of current, which is expressed by

$$
\mathbf{i}=F \sum_{k=1}^{M} z_{k} \mathbf{N}_{k}=F \sum_{k=1}^{M} z_{k}\left[\left(1-\sigma_{k}\right) C_{k} \mathbf{q}-\overline{\boldsymbol{\omega}}_{k} R T\left(\nabla C_{k}+\frac{z_{k} F}{R T} C_{k} \nabla \Phi\right)\right]=0
$$

where $\mathbf{i}$ is the current density. Equation (23) can be used to determine the gradient of the electrostatic potential. Substituting equations (21) and (22) into (14) and (15), yields,

$$
\frac{\partial J}{\partial t}=\nabla_{o} \cdot\left[\mathbf{K} \nabla p-\mathbf{K} \sum_{k=1}^{M} \sigma_{k} R T\left(\nabla C_{k}+\frac{z_{k} F}{R T} C_{k} \nabla \Phi\right)\right]
$$




$$
\begin{aligned}
& \frac{\partial}{\partial t}\left[\left(J-1+\phi_{o}\right) C_{k}\right]=\nabla_{o} \cdot\left[\mathbf{D}_{k}\left(\nabla C_{k}+\frac{z_{k} F}{R T} C_{k} \nabla \Phi\right)\right]+ \\
& \nabla_{o} \cdot\left\{\left(1-\sigma_{k}\right) C_{k} \mathbf{K}\left[\nabla p-\sum_{k=1}^{M} \sigma_{k} R T\left(\nabla C_{k}+\frac{z_{k} F}{R T} C_{k} \nabla \Phi\right)\right]\right\}
\end{aligned}
$$

where $\mathbf{D}_{k}=\overline{\mathbf{\omega}}_{k} R T, \mathbf{K}=\overline{\mathbf{L}}_{p}$, and $\zeta=J-1$ have been applied. Equations (5), (6), (7), (24) and (25) are the governing equations for determining stresses, strains, displacements, fluid pressure, and ionic concentrations in ionic solution-filled porous materials. Unlike the biphasic model where only the interaction between the solid and fluid is considered, the above model involves the interactions between the solid, ionic solution and ionic species, and so is referred to as the triphasic model. If finite element methods are used to solve the coupled system, the primary variables can be taken to be the displacements of the matrix $u$, the variation of fluid content $\zeta$, and the concentrations of ionic species $\mathrm{C}_{\mathrm{k}}$; all other quantities can be expressed in terms of these primary variables.

\section{Application of the triphasic model in prediction of corneal swelling}

As an application of the proposed triphasic model, we consider a case where a human cornea is subject to an osmotic perturbation of $\mathrm{NaCl}$ solution applied to its endothelial surface. Assume that the tissue is initially equilibrated with the external bathing solution. Thus an increase in concentration in the bathing solution will cause an outward flow from the tissue and thus a reduction of the corneal thickness. For simplicity, we assume that the deformation is small and the strains parallel to the corneal surface are negligible and thus the problem can be simplified into a onedimensional problem in which all unknowns are only a function of the coordinate along the thickness direction. In this case, the strain-displacement relationship (5), equilibrium equation (6) and constitutive equation (7) become

$$
\begin{aligned}
& \varepsilon_{x x}=\frac{d u}{d x} \\
& \sigma_{x x}=\text { constant } \\
& \left(\sigma_{x x}-\sigma_{o}\right)=(\lambda+2 \mu) \varepsilon_{x x}-p
\end{aligned}
$$

where $u$ is the displacement, $\varepsilon_{x x}$ is the strain, $\sigma_{x x}$ is the total stress, $\sigma_{o}$ is the initial stress which is generated by the swelling pressure $p_{s w}, \lambda$ and $\mu$ are the Lame constants. The swelling pressure can be expressed in terms of the stromal hydration $H_{w}$ which is defined as the mass of water in unit mass of dry tissue material. Hence, the initial stress can be expressed as $\sigma_{o}=-p_{s w}=-2.41 \times 10^{6} \exp \left(-H_{w}\right)$ in dyn- $\mathrm{cm}^{-2}$ ( $\mathrm{Li}$ and Tighe, 2006). The normal thickness of the cornea is about $0.5 \mathrm{~mm}$, the maximum increase or decrease of the corneal thickness in a perfusion experiment is only about $20 \mu \mathrm{m}$ ( $\mathrm{Li}$ and Tighe, 200), which gives a maximum strain of $4 \%$. This justifies the above assumptions of small deformation and linear version of constitutive equations when considering that the matrix is a soft material which has very low elastic modulus. The inclusion of swelling pressure in equation (28) is based on the fact that, in the absence of fluid pressure, an external compression stress must be applied in order to 
prevent the stromal swelling. Based on the stress boundary condition at the endothelial surface the stress constant in equation (27) can be identified, which in value is equal to the intraocular pressure $\left(\mathrm{IOP}=2.67 \times 10^{4} \mathrm{dyn}-\mathrm{cm}^{-2}\right)$ but in an opposite direction ( $\mathrm{Li}$ and Tighe, 2006). Thus, from equations (27) and (28) the fluid pressure can be expressed as follows

$$
p=(\lambda+2 \mu) \varepsilon_{x x}+I O P-p_{s w}
$$

Equation (29) implies that the strain-induced stress plus IOP will balance the swelling pressure and the fluid pressure. It is obvious that if the elastic stiffness of the tissue is very low, then the first term in the right-hand-side of equation (29) may be negligible. In this case the fluid pressure is only dependent on the IOP and swelling pressure, and therefore the tissue deformation can be determined purely from the transport model of ions and ionic solution which has been studied by several researchers (Klyce and Russell, 1979; Li et al., 2004; Li and Tighe, 2006). Assume that the reflection coefficients for chloride and sodium ions are identical, i.e., $\sigma_{\mathrm{Cl}}=\sigma_{\mathrm{Na}}=\sigma_{\mathrm{kk}}$. By using electro-neutrality condition (i.e., $\mathrm{C}_{\mathrm{Cl}}=\mathrm{C}_{\mathrm{Na}}=\mathrm{C}$ ), equation (23) can be simplified as

$$
\frac{F}{R T} \frac{\partial \Phi}{\partial x}=-\frac{z_{C l} D_{C l} \frac{\partial C_{C l}}{\partial x}+z_{N a} D_{N a} \frac{\partial C_{N a}}{\partial x}}{z_{C l}^{2} D_{C l} C_{C l}+z_{N a}^{2} D_{N a} C_{N a}}=\frac{\left(D_{C l}-D_{N a}\right)}{\left(D_{C l}+D_{N a}\right)} \frac{1}{C} \frac{\partial C}{\partial x}
$$

where $z_{N a}=1$ and $z_{C l}=-1$ are the charge numbers of $\mathrm{Na}$ and $\mathrm{Cl}$ ions, $D_{\mathrm{Na}}$ and $D_{\mathrm{Cl}}$ are the diffusion coefficients of $\mathrm{Na}$ and $\mathrm{Cl}$ ions. Using equation (30), equations (24) and (25) can be simplified as

$$
\begin{aligned}
& \frac{\partial \varepsilon_{x x}}{\partial t}=k_{x x}\left(\frac{\partial^{2} p}{\partial x^{2}}-2 \sigma_{k k} R T \frac{\partial^{2} C}{\partial x^{2}}\right) \\
& \frac{\partial}{\partial t}\left[\left(\varepsilon_{x x}+\phi_{o}\right) C\right]=\frac{2 D_{N a} D_{C l}}{D_{N a}+D_{C l}} \frac{\partial^{2} C}{\partial x^{2}}+\left(1-\sigma_{k k}\right) k_{x x} C\left(\frac{\partial^{2} p}{\partial x^{2}}-2 \sigma_{k k} R T \frac{\partial^{2} C}{\partial x^{2}}\right)
\end{aligned}
$$

where $k_{x x}$ is the permeability coefficient in the thickness direction. Using equation (31) to simplify (32), it yields,

$$
\left(\varepsilon_{x x}+\phi_{o}\right) \frac{\partial C}{\partial t}=\frac{2 D_{N a} D_{C l}}{D_{N a}+D_{C l}} \frac{\partial^{2} C}{\partial x^{2}}-\sigma_{k k} k_{x x} C\left(\frac{\partial^{2} p}{\partial x^{2}}-2 \sigma_{k k} R T \frac{\partial^{2} C}{\partial x^{2}}\right)
$$

Equations (29), (31) and (33) are the three equations used to solve for the fluid pressure, strain, and ionic concentration. Note that the variation of the stromal hydration is related to the strain. The relationship between the two is as follows,

$$
\varepsilon_{x x}=\frac{\left(H_{w}-H_{w o}\right) / \rho_{w}}{H_{w o} / \rho_{w}+1 / \rho_{d}}=\frac{H_{w}-H_{w o}}{H_{w o}+\rho_{w} / \rho_{d}}
$$

where $\rho_{w}=1 \mathrm{~g}-\mathrm{cm}^{-3}$ is the density of water, $\rho_{d}=1.49{\mathrm{~g}-\mathrm{cm}^{-3}}^{-3}$ the density of the dry tissue material, and $H_{w o}=3.4$ is the initial stromal hydration. For given initial condition 
(stromal hydration $H_{w o}$ and ionic concentration $C_{o}$ ) and boundary conditions (fluid pressure $p$ or $d p / d x$ and ionic concentration $C$ or $d C / d x$ ), one can solve the partial differential equations (31) and (33) where the fluid pressure and stromal hydration can be expressed in terms of the strain by using equations (29) and (34). It should be pointed out that although the governing equations (29) and (31) for the solid and fluid phases are linear, the transport equation (33) for the ionic species is nonlinear due to the involvement of the change of porosity. Therefore, the coupled system of equations is not only time-dependent but also nonlinear.

Figure 3 shows a typical example of the corneal response to the shock of $\mathrm{NaCl}$ hypertonic solution applied (after the steady state has been reached) to the endothelial surface for a hour duration, while the epithelial surface of the cornea is left intact. The transport parameters employed here are given in Table 1 which are the same as those used in $\mathrm{Li}$ and Tighe $(2004,2006 \mathrm{a})$. The only difference is the equation (29) for fluid pressure; here it is also dependent on the volume dilatation of the tissue, while in $\mathrm{Li}$ and Tighe $(2004,2006 a) \lambda+2 \mu \equiv 0$ was assumed. The Lame constants for a highly hydrated proteoglycan-containing matrix are estimated as $\lambda+2 \mu \approx 15 \mathrm{kPa}$ (Pinsky and Datye, 1991; Hjortdal, 1996; Li and Tighe, 2006b). In practice, however, the value of $\lambda+2 \mu$ in the thickness direction would be greater than $15 \mathrm{kPa}$ because of the lamellae interlacing. Therefore, it is thought to be more reasonable to use $\lambda+2 \mu=20$ $\mathrm{kPa}$ in the example. In order to show the influence of the elastic properties of the tissue material on its swelling in the collagen fibril direction, a large value of $\lambda+2 \mu=100 \mathrm{kPa}$ is also used. The plotted results show that the thickness changes to the shock are smaller when the strain-induced stress is included in the model. This is to be expected. As the tissue shrinks (or swells) due to the outward (or inward) volumetric flow, the solid matrix of the tissue will provide a "spring-like" mechanical resistance. This mechanical resistance will produce an opposite direction strain, which leads the overall strain be reduced. The results shown in figure 3 indicate that, the larger the value of $\lambda+2 \mu$, the smaller the changes in thickness. It is expected that, when the value of $\lambda+2 \mu$ becomes large enough so that the mechanical stress and the swelling pressure are in balance, there will be no change in the thickness.

It should be mentioned that the example shown here only demonstrates the influence of the elastic modulus of the matrix material on the response of the corneal thickness to the perfusion of external solutions. The interaction feature involving the nonlinear material characteristics of the cornea has to be investigated through a full threedimensional model which is currently being developed. Also, it should be pointed out that, because of the lack of experimental data all material constants used here in the example are the same as those used in the previous transport models (see, for example, Klyce and Russell, 1979; Li and Tighe, 2006a). Since most of these material constants were determined by using a best fit between the transport model, which did not consider the influence of solid phase, and the perfusion experiments, this makes it difficult to validate the present model. Nevertheless, the present results have clearly shown that if the solid phase influence is considered the corneal thickness response will be reduced remarkably. This suggests that the previously determined material constants need be re-assessed. It is noted than even for the one-dimensional model considered here, there are about 20 material constants that are required to determine.

\section{Conclusions}


A nonlinear, macroscopic multi-phasic model has been proposed for describing the interactions between solid, fluid, and ionic species taking place in porous materials. Governing equations have been derived based on nonlinear theories of solid mechanics, linear flow theory of Newtonian fluids, and the theory of irreversible thermodynamics for the transport of ions and ionic solution. The model is built up on Biot's poroelasticity but considers the nonlinearity of the porous material and the influence of transport of ionic species on the flow of ionic solution and the deformation of the porous material.

The present model has shown that the coupling between solid, fluid and ionic species exists when the porous material involves membranes or has membrane-like features, which could be inside or just on the boundaries of the material. Otherwise, the coupling occurs only between the solid and fluid phases in which case the problem can be solved using the biphasic model.

As the application of the present multi-phasic model, a numerical example of the human cornea under the shock of $\mathrm{NaCl}$ hypertonic solution applied to its endothelial surface has been investigated. The results have demonstrated that the mechanical properties of the tissue have important influence on the swelling of the cornea. Without taking into account this influence, the predicted swelling could be exaggerated.

Acknowledgement - This work was supported by the UK EPSRC research grant (EP/G056501/1).

\section{References}

Bear J. Dynamics of Fluids in Porous Media. 1988, Dover Publication, New York.

Biot M.A. General theory of three-dimensional consolidation. Journal of Applied Physics 12, 1941, 155-164.

Biot M.A. The elastic coefficients of the theory of consolidation. Journal of Applied Mechanics (ASME) 24, 1957, 594-601.

Biot M.A. Nonlinear and semi-linear rheology of porous solids. Journal of Geophysical Research 78, 1973, (23)4924-4937.

Cohen B., Lai W.M. and Mow V.C. A transversely isotropic biphasic model for unconfined compression of growth plate and chondroepiphysis. Journal of Biomechanical Engineering 120, 1998, 491-496.

De Bore R. Theory of Porous Media: Highlights in the Historical Development and Current State, 2000, Springer, Berlin.

Detournay E. and Cheng A.H.D. Fundamental of poroelasticity. In: Fairhurst C. (ed.): Comprehensive Rock Engineering, Principles, Practice and Projects. Vol.II, Analysis and Design Method. Pergamon Press, Oxford, 1993, 113-171.

Ferguson S.J., Ito K. and Nolte L.P. Fluid flow and convective transport of solutes within the intervertebral disc. Journal of Biomechanics 37, 2004, 213-221.

Gasser T.G., Ogden R.W. and Holzapfel G.A. Hyperelastic modelling of arterial layers with distributed collagen fibre orientations. Journal of the Royal Society Interface 3, 2006, (6)15-35. 
Gu W.Y., Lai W.M. and Mow V.C. Transport of fluid and ions through a porouspermeable charged-hydrated tissue, and streaming potential data on normal bovine articular cartilage. Journal of Biomechanics 26, 1993, 709-723.

Gu W.Y., Lai W.M. and Mow V.C. A triphasic analysis of negative osmotic flows through charged hydrated soft tissues. Journal of Biomechanics 30, 1997, 71-78.

$\mathrm{Gu}$ W.Y., Lai W.M. and Mow V.C. Transport of multi-electrolytes in charged hydrated biological soft tissues. Transport in Porous Media 34, 1999, 143-157.

Hjortdal J.O. Regional elastic performance of the human cornea. Journal of Biomechanics 29, 1996, (7)931-942.

Holmes M.H. Finite deformation of soft tissue: analysis of a mixture model in uniaxial compression. Journal of Biomechanical Engineering 108, 1986, (4)372-382.

Huyghe J. and Janssen J.D. Thermo-chemo-electro-mechanical formulation of saturated charged porous solids. Transport in Porous Media 34, 1999, (1-3)129-141.

Kedem O. and Katchalsky A. Thermodynamic analysis of the permeability of biological membranes to non-electrolytes. Biochim. Biophys. Acta 27, 1958, 229246.

Klyce S.D. and Russell S.R. Numerical solution of coupled transport equations applied to corneal hydration dynamics. Journal of Physiology (London) 292, 1979, 107-134.

Lai W.M., Hou J.S. and Mow V.C. A triphasic theory for the swelling and deformation behaviours of articular cartilage. Journal of Biomechanical Engineering 113, 1991, 245-258.

Li L.Y. Transport of multicomponent ionic solutions in membrane systems. Philosophical Magazine Letter 84, 2004, (9)593-599.

Li L.Y., Tighe B.J. and Ruberti J.W. Mathematical modelling of corneal swelling. Biomech. Model. Mechanobiol. 3, 2004, 114-123.

Li L.Y. and Tighe B.J. Numerical simulation of corneal transport processes. Journal of The Royal Society Interface 3, 2006a, 303-310.

Li L.Y. and Tighe B.J. The anisotropic material constitutive models for the human cornea. Journal of Structural Biology 153, 2006b, 223-230.

Lopatnikov S.L. and Cheng A.H.D. Variational formulation of fluid infiltrated porous material in thermal and mechanical equilibrium. Mechanics of Materials 34, (2002), 685-704.

Mak A.F.T. and Huang L. A biphasic poroelastic analysis of the flow dependent subcutaneous tissue pressure and compaction due to epidermal loadings: issues in pressure sore. Journal of Biomechanical Engineering 116, 1994, (4)421-430.

Mak A.F.T. and Zhang J.D. Numerical simulation of streaming potentials due to deformation-induced hierarchical flows in cortical bone. Journal of Biomechanical Engineering 123, 2001, (1)66-70.

Mow V.C., Holmes M.H. and Lai W.M. Fluid transport and mechanical properties of articular cartilage: a review. Journal of Biomechanics 17, 1984, (5)377-394.

Mow V.C., Kuei S.C., Lai W.M. and Armstrong C.G. Biphasic creep and stress relaxation of articular cartilage in compression: theory and experiments. Journal of Biomechanical Engineering 102, 1980, (1)73-84.

Naili S., Oddou C. and Geiger D. A method for the determination of mechanical parameters in a porous elastically deformable medium: applications to biological soft tissues. Int. J. Solids Structures 35, 1998, 4963-4979.

Newman J.S. Electrochemical Systems ( $2^{\text {nd }}$ edition), 1973. Prentice-Hall, Englewood Cliffs, New Jersey. 
Pinsky P.M. and Datye D.V. A microstructurally based finite element model of the incised human cornea. Journal of Biomechanics 24, 1991, 907-922.

Pinsky P.M., van der Heide D. And Chernyak D. Computational modeling of mechanical anisotropy in the cornea and sclera. Journal Cataract Refract. Surg. 31, 2005, 136-145.

Simon B.R., Kaufmann M.V. and McAfee M.A. Finite element models for arterial wall mechanics. Journal of Biomechanical Engineering 115, 1993, (4B)489-497.

Simon B.R., Kaufman M.V., Liu J. and Baldwin A.L. Porohyperelastic-transportswelling theory, material properties and finite element models for large arteries. International Journal of Solids and Structures 35, 1998, (34/35)5021-5031.

Snijders H., Huyghe J.M. and Janssen J.D. Triphasic finite element model for swelling porous media. International Journal for Numerical Methods in Fluids 20, 2005, (8/9)1039 - 1046.

Soltz M.A. and Ateshian G.A. A conewise linear elasticity mixture model for the analysis of tension-compression nonlinearity in articular cartilage. Journal of Biomechanical Engineering 122, 2000, 576-586.

Soulhat J., Buschmann M.D. and Shirazi-Adl A. A fibril-network-reinforced biphasic model of cartilage in unconfined compression. Journal of Biomechanical Engineering 121, 1999, 340-347.

Swartz M.A., Kaipainen A., Netti P.A., Brekken C., Boucher Y., Grodzinsky A. and Jain R.K. Mechanics of interstitial-lymphatic fluid transport: theoretical foundation and experimental validation. Journal of Biomechanics 32, (1999), 1297-1307.

Terzaghi K. Theoretical Soil Mechanics, 1943, John Wiley and Sons, New York.

van Meerveld J., Molenaar M.M., Huyghe J.M. and Baaijens F.P.T. Analytical solution of compression, free swelling and electrical loading of saturated charged porous media. Transport in Porous Media 50, 2003, (1/2) 111-126.

Yang M. and L.A. Taber L.A. The possible role of poroelasticity in the apparent viscoelastic behavior of passive cardiac muscle. Journal of Biomechanics 24, 1991, (7) 587-597.

Zhang L. and Szeri A.Z. Transportation of neutral solute in deformable, anisotropic, soft tissue. Computers \& Mathematics with Applications 53, 2007, (2)232-243.

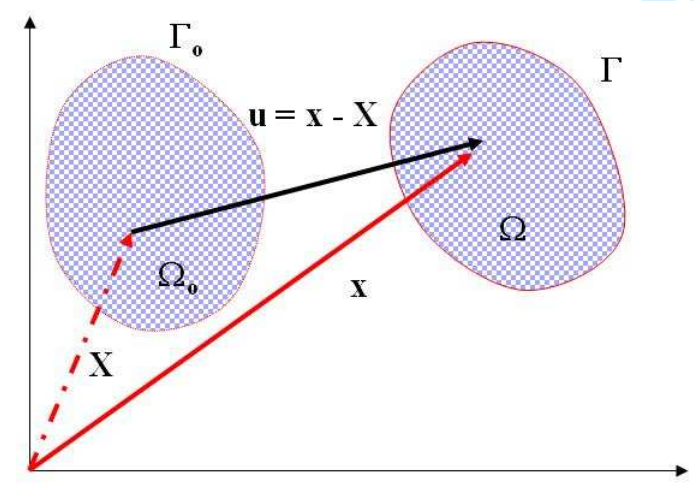

Figure 1. Configurations of undeformed and deformed porous material. 


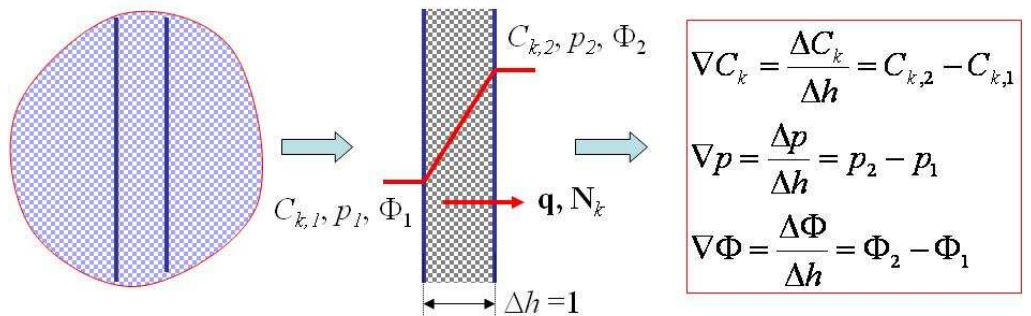

Figure 2. Sign definition of fluxes in membrane wall (arrow direction represents q and $\mathrm{N}$ being positive).

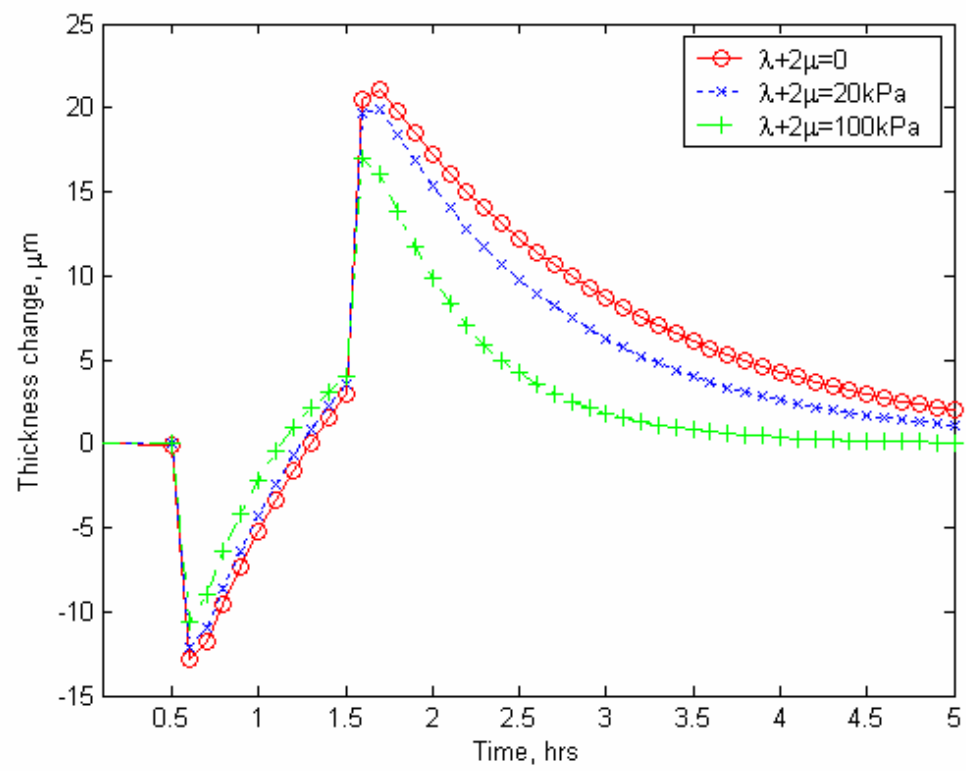

Figure 3. Stromal thickness response to the shock of $15 \mathrm{mOsm} \mathrm{NaCl}$ hypertonic solution applied (after a steady state) to the endothelial surface for an hour duration while the epithelial surface is left intact. 
Table 1. Parametric values employed in the numerical studies for figure 3 ( $\mathrm{Li}$ and Tighe, 2004, 2006a).

\begin{tabular}{|c|c|c|c|}
\hline Parameter & $\mathrm{Na}^{+}$ & $\mathrm{Cl}^{-}$ & $P$ \\
\hline Charge number & 1 & -1 & - \\
\hline Molar mass & $11 \mathrm{~g} / \mathrm{mole}$ & $17 \mathrm{~g} / \mathrm{mole}$ & - \\
\hline $\begin{array}{l}\text { Initial } \\
\text { conditions }\end{array}$ & $145 \times 10^{-6} \mathrm{~mole} / \mathrm{cm}^{3}$ & $145 \times 10^{-6} \mathrm{~mole} / \mathrm{cm}^{3}$ & $\begin{array}{l}2.67 \times 10^{4} \\
\text { dyne } / \mathrm{cm}^{2}\end{array}$ \\
\hline $\begin{array}{l}\text { BCs at } \\
\text { epithelium }\end{array}$ & $\mathrm{dC} / \mathrm{dx}=0$ & $\mathrm{dC} / \mathrm{dx}=0$ & $\mathrm{dp} / \mathrm{dx}=0$ \\
\hline $\begin{array}{l}\text { BCs at } \\
\text { endothelium }\end{array}$ & $\begin{array}{c}(145+15) \times 10^{-6} \\
\text { mole } / \mathrm{cm}^{3}\end{array}$ & $\begin{array}{c}(145+15) \times 10^{-6} \\
\text { mole } / \mathrm{cm}^{3}\end{array}$ & $\begin{array}{l}2.67 \times 10^{4} \\
\text { dyne } / \mathrm{cm}^{2}\end{array}$ \\
\hline $\begin{array}{l}\text { Permeability } \\
\text { coefficient in } \\
\text { epithelium }\end{array}$ & & $\begin{array}{l}2.90 \times 10^{-15} \\
\mathrm{~cm}^{4} /(\text { s-dyne })\end{array}$ & \\
\hline $\begin{array}{l}\text { Permeability } \\
\text { coefficient in } \\
\text { stroma }\end{array}$ & $P=$ & $\begin{array}{l}.63 \times 10^{-15} \mathrm{H}_{\mathrm{w}}{ }^{4} \\
\mathrm{~cm}^{4} /(\mathrm{s}-\text { dyne })\end{array}$ & \\
\hline $\begin{array}{l}\text { Permeability } \\
\text { coefficient in } \\
\text { endothelium }\end{array}$ & & $\begin{array}{c}2.65 \times 10^{-15} \\
\mathrm{~cm}^{4} /(\mathrm{s}-\text { dyne })\end{array}$ & \\
\hline $\begin{array}{l}\text { Reflection } \\
\text { coefficient in } \\
\text { epithelium, } \sigma_{k k}\end{array}$ & 0.800 & 0.580 & - \\
\hline $\begin{array}{l}\text { Reflection } \\
\text { coefficient in } \\
\text { stroma, } \sigma_{k k}\end{array}$ & 0 & 0 & - \\
\hline $\begin{array}{l}\text { Reflection } \\
\text { coefficient in } \\
\text { endothelium, } \sigma_{k k}\end{array}$ & 0.685 & 0.450 & - \\
\hline $\begin{array}{l}\text { Diffusion } \\
\text { coefficient in } \\
\text { epithelium, D }\end{array}$ & $\begin{array}{c}4.95 \times 10^{-10} \\
\mathrm{~cm}^{2} / \mathrm{s}\end{array}$ & $\begin{array}{c}7.5 \times 10^{-10} \\
\mathrm{~cm}^{2} / \mathrm{s} \\
\end{array}$ & - \\
\hline $\begin{array}{l}\text { Diffusion } \\
\text { coefficient in } \\
\text { stroma, D }\end{array}$ & $\frac{5.91 \times 10^{-6} H_{w}}{H_{w}+\rho_{w} / \rho_{d}} \mathrm{~cm}^{2} / \mathrm{s}$ & $\frac{9.0 \times 10^{-6} H_{w}}{H_{w}+\rho_{w} / \rho_{d}} \mathrm{~cm}^{2} / \mathrm{s}$ & - \\
\hline $\begin{array}{l}\text { Diffusion } \\
\text { coefficient in } \\
\text { endothelium, D }\end{array}$ & $\begin{array}{c}2.63 \times 10^{-8} \\
\mathrm{~cm}^{2} / \mathrm{s}\end{array}$ & $\begin{array}{c}4.00 \times 10^{-8} \\
\mathrm{~cm}^{2} / \mathrm{s}\end{array}$ & 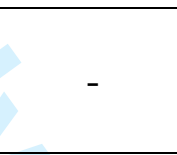 \\
\hline $\begin{array}{l}\text { Active pump } \\
\text { rate at } \\
\text { endothelium, } J\end{array}$ & 0 & $\begin{array}{c}-4.0 \times 10^{-10} \\
\left.\text { mole/(s-cm }{ }^{2}\right)\end{array}$ & - \\
\hline
\end{tabular}




\section{Dear Editor}

Thank you very much for sending me the valuable comments and suggestions made by the three reviewers. We have revised our paper in the light of these comments and suggestions and the following are the responses (text with blue colour) to the reviewers' comments and the new texts added in the revised paper are highlighted with yellow colour.

\section{Referee: 1}

"The some derived equations in Section 2 and Section 3 have nonlinear expressions, but the discussion in Section 4 basically made them to be linear expressions. Hence Figure 3 is similar to that of Li and Tighe $(2004,2006 a)$, which the nonlinear characteristics focused on was not displayed completely."

There are three sets of governing equations (each phase has one set). Because of the coupling, governing equations in liquid and ionic species phases are also nonlinear. Only simplification used in the example section is the governing equations in solid phase which are linearised, while in sections 2 and 3 they are nonlinear. This simplification is purely because the use of one-dimensional model (normal to the thickness), in which case the elastic modulus is only due to the matrix material (which is almost linear) and the collagen fibrils have no contribution to the modulus of elasticity. The main purpose of Fig. 3 is not to show the nonlinear characteristics but to demonstrate how the elastic modulus can affect the thickness change during a numerical perfusion experiment. These two points have been re-addressed in the text of the example section (see added text in pages 9 and 11). It should be pointed out that Li and Tighe (2004, 2006a) model did not have the solid phase while the present model including the example has although in the example only linear equations of the solid phase are considered (see also our response 3 to Referee 2).

"Journal readers may not have more profound physical or mechanical basis, and are not good at understanding complex formula. If it is allowed, the manuscript may give some clearer example of verification, then the readers can easily understand."

See our response to Referee 3.

\section{Referee: 2}

1) Some of the equations in section 2 are textbook material and can be taken out by referring to a textbook.

It is true that Eqs.(2)-(6) can be found in some high level textbooks. However, they are used a few times during the derivation of other equations in sections 3 and 4 and therefore it is better to keep them. If they were removed it would be difficult for readers to follow.

2) It would be useful to point out what are the basic field variables (such as displacement in an elasticity problem) that need to be solved for after Eqn. (25).

These have been added in text (see page 9). 
3) In section 4, a justification for the small deformation assumption would be helpful, what is the actual level of deformation during the corneal swelling?

The normal thickness of the cornea is about $0.5 \mathrm{~mm}$. The increase or decrease of the thickness during a perfusion experiment is about 20 micro-meters (see Fig.3), which gives the maximum strain of about $4 \%$. Thus small deformation assumption is justified. This is particularly true for the matrix material which has small elastic modulus. Some text has been added in section 4 (page 9) to justify this.

4) References should be provided for the source of data in Table 1

This has been added in text (see page 16).

5) Fig 2, sign definition - sign convention.

This has been added in text (see page 14).

\section{Referee: 3}

"The governing equations of the model seem correct to me, but it would be helpful if the authors included a plot, table, or at least a sentence about validation. The previous model (Li and Tighe, 2006a) was able to reproduce experimental data (Fischbarg, J. 1973, Exp. Eye Res. 15, 615-638.) very well and there did not seem to be much room for further improvement (in terms of agreement with experiment). It would be interesting to see how the present model compares with the same or other experiment. This might help readers decide whether to adopt the presented model.

The main purpose of this paper is to demonstrate how important the interaction between solid, liquid and ionic species phases is. As it can be seen from the present tri-phase model, there are two sets of material constants, one for the transport of solution and solutes and the other for the mechanical properties. Currently, the constants for the transport were determined based on the best fit between the transport model which did not consider the solid phase influence and the perfusion experiments (see, for example, Klyce and Russell, 1979). This is why "The previous model (Li and Tighe, 2006a) was able to reproduce experimental data (Fischbarg, J. 1973, Exp. Eye Res. 15, 615-638.) very well'. The present results show that if the solid phase influence is considered the corneal thickness response will be reduced remarkably. This suggests that the previously determined constants need be re-assessed. Since even for the one-dimensional model there are still about 20 material constants required to determine, this requires more experimental data and is obviously out of the scope of this paper. More explanation has been added in text (see pages 11/12)

The manuscript is organized in a logical way and is written in good English. I have noticed some minor typographical errors:

1) In abstract: "typical example of ..." instead of "typical example about ..."

2) Section 4, last paragraph: "Lame constants" instead of "lame constants". Better yet "Lamé constants"

These errors have been corrected. 